\title{
Phase dynamics of delay-coupled quasi-cycles with application to brain rhythms
}

\author{
Arthur S. Powanwe $\mathbb{0}^{*}$ and André Longtin \\ Physics Department, University of Ottawa, 150 Louis Pasteur, Ottawa, Ontario, Canada K1N 6N5
}

(Received 1 May 2020; accepted 15 September 2020; published 13 October 2020)

\begin{abstract}
We consider the phase locking of two delay-coupled quasi-cycles. A coupled envelope-phase system obtained via stochastic averaging enables a stability analysis. While for deterministic limit-cycle oscillators the coupling can produce in-phase, antiphase, and the intermediate "out-of-phase" locking (OPL) behavior via spontaneous symmetry breaking, such outcomes for the quasi-cycle case are shown to require instead both noise and coupling delay. The theory, which applies the stochastic averaging method to delayed dynamics, generates stochastic stability functions that predict the numerically observed OPL behavior as a function of all the system parameters. OPL for coupled quasi-cycles occurs for additive or multiplicative noise, and for coupled networks of excitatory and inhibitory neurons as well as networks of inhibitory neurons coupled to one another. Our theory also predicts that the bifurcation at which the in-phase state becomes unstable lies at smaller delays for stronger noise. The noise produces the realistic quasi-cycle rhythms and out-of-phase behavior, all the while causing random reversals of the phase leader. Asymmetry in the coupling between networks, as well as heterogeneity within each network, also allows for quasi-cycle OPL, although it produces asymmetric bifurcations that bias the leadership towards one of the networks. These results are relevant to communication between brain areas and other networks that rely on noise-induced rather than noise-perturbed rhythms.
\end{abstract}

DOI: 10.1103/PhysRevResearch.2.043067

\section{INTRODUCTION}

Noisy oscillations are observed in a wide variety of systems. Our understanding of the behavior of these systems in isolation and in networks is governed by the underlying dynamical origin of the oscillation. The case in which the system possesses a deterministic limit cycle which is perturbed by noise has received much attention [1-3]. Much is known about the synchronization properties of noise-perturbed limit cycle systems, such as their ability to lock in phase (IP), in antiphase (AP), or at phase differences in between, i.e., out-of-phase locking (OPL). In contrast, there may be no underlying deterministic limit cycle, and the generation of the observed oscillations relies on noise. Although studied to a much lesser extent, these noise-induced oscillations, known as "quasi-cycles," arise in an increasing number of contexts, from semiconductor lasers to brain dynamics and epidemics [4-7]. Theory is particularly lacking for coupled quasi-cycles, thought to enable, e.g., oscillation (or "rhythm")-based communication between brain areas [7,8].

In this work, the stochastic averaging method (SAM) is used to obtain an envelope-phase description for coupled quasi-cycles. It reveals a novel mechanism for OPL. Delays are included as they are often non-negligible compared to other system time scales $[9,10]$.

\footnotetext{
*apowa074@uottawa.ca
}

Published by the American Physical Society under the terms of the Creative Commons Attribution 4.0 International license. Further distribution of this work must maintain attribution to the author $(s)$ and the published article's title, journal citation, and DOI.
Although the findings below apply generally, we are particularly motivated by the communication between brain areas, thought to require the phase difference between two rhythms in separate areas being constant over some time interval [11]. The sign of this difference reveals the leadership and direction of the information flow between the areas [12]. For identical, symmetrically coupled networks the available theory, based on deterministic oscillators, invokes spontaneous symmetry breaking (SSB) to explain OPL. SSB is a deterministic effect where the two-oscillator system loses symmetry beyond a critical parameter value, with one becoming the leader and the other the laggard $[13,14]$. Flexible, reversible bidirectional communication can then be achieved with brief external pulses that reverse the leadership [15-17].

However, there is increasing evidence that many brain rhythms are noise induced and manifest only in brief epochs of random duration $[8,10,18,19]$. OPL can also be seen, but phase locking of quasi-cycles is not well understood, a specific motivator for our work. While SSB is here shown to not occur for coupled quasi-cycles, OPL can arise thanks both to delays and the smoothing by noise of the bifurcation between IP and AP locking.

Two dominant mechanisms for rhythms in the higher "gamma" frequency range have been reported in the experimental and theoretical neuroscience literature: Pyramidalinterneuron network gamma, which involves reciprocally and self-coupled excitatory and inhibitory neurons (E-I networks), and interneuron network gamma, which sees inhibitory neurons coupled to one another (I-I networks), often with a delay. The mechanism of delay and noise-induced OPL in coupled quasi-cycles is shown below to be present in two coupled E-I networks (our main focus), as well as in two coupled I-I networks. In both cases, this OPL persists even if the networks 
are heterogeneous but symmetrically coupled, or identical but asymmetrically coupled. This suggests a strong and robust mechanism which could underlie some of the dynamic functional connectivity observed in the brain of several species at rest and when performing certain tasks [20-22]. These coupled dynamics, in which each network exhibits quasi-cycle behavior in isolation, also enable bidirectional exchanges of information between networks [8]. The leadership can then alternate randomly between the two networks following fluctuations in the random inputs, as well as be biased by external pulses as in the case of the coupled oscillator dynamics. We thus extend to noise-induced rhythms the property of communication through coherence $[23,24]$ and its expanded version with propagation delay [25].

After presentation of the general PING model with noise, we derive the envelope-phase dynamics by extending the SAM technique to delayed coupling. Analysis of the symmetric case reveals the delay-and-noise-induced OPL phenomenon in the coupled quasi-cycles. This is followed by an extension to the asymmetric and heterogeneous cases. We complete our study of coupled quasi-cycles by considering the alternative ING model in the same cases, and end with a discussion and outlook onto future works.

\section{MODEL OF COUPLED E-I NETWORKS}

We are motivated by the work on stochastic E-I networks in Ref. [26], in which all neurons in each population are simulated as two-state systems that are either quiescent or active. This leads to a two-dimensional Wilson-Cowan-type rate model with an $\mathrm{E}$ and an $\mathrm{I}$ population each with their own finite size fluctuations. This formalism can also model sparse connectivity through an appropriate scaling of the mean synaptic coupling coefficients. To set the scene, we describe their model in the microscopic, multineuron context with specific neuron numbers. However, all the analytical and numerical work is done on the associated two-variable model.

Each of the two networks has $N_{E}$ excitatory $(E)$ and $N_{I}$ inhibitory $(I)$ neurons with $N_{E}=4 N_{I}$, coupled to produce oscillations near but below a Hopf bifurcation. These two networks are further symmetrically coupled via long-range excitatory connections with propagation delay as in Fig. 1. The state variables are the mean activity of the neurons in each population, i.e., $E_{i}$ and $I_{i}, i=1,2$. The coefficients $W_{E E}$ and $W_{I I}$ are respectively the recurrent excitatory and inhibitory synaptic coupling, while $W_{I E}$ and $W_{E I}$ are, respectively, the feedback excitatory and inhibitory synaptic coupling in each network. In addition, each population in each network receives a constant external input ( $h_{E}$ and $h_{I}$, respectively). The long-range excitatory synaptic coupling between the two E populations is $L_{E E}$, and between the E population of one network and the I population of the other network is $L_{I E}$. The activity of a neuron in one network is felt by the other network after a delay $\tau$.

The model is schematized in Fig. 1(a) and described by the stochastic Wilson-Cowan (SWC) equations [26]:

$$
\begin{aligned}
\dot{E}_{i}(t) & =-\alpha_{E} E_{i}(t)+\left[1-E_{i}(t)\right] \beta_{E} f\left(s_{E_{i}}(t)\right)+g_{1} \xi_{E_{i}}(t), \\
\dot{I}_{i}(t) & =-\alpha_{I} I_{i}(t)+\left[1-I_{i}(t)\right] \beta_{I} f\left(s_{I_{i}}(t)\right)+g_{2} \xi_{I_{i}}(t),
\end{aligned}
$$
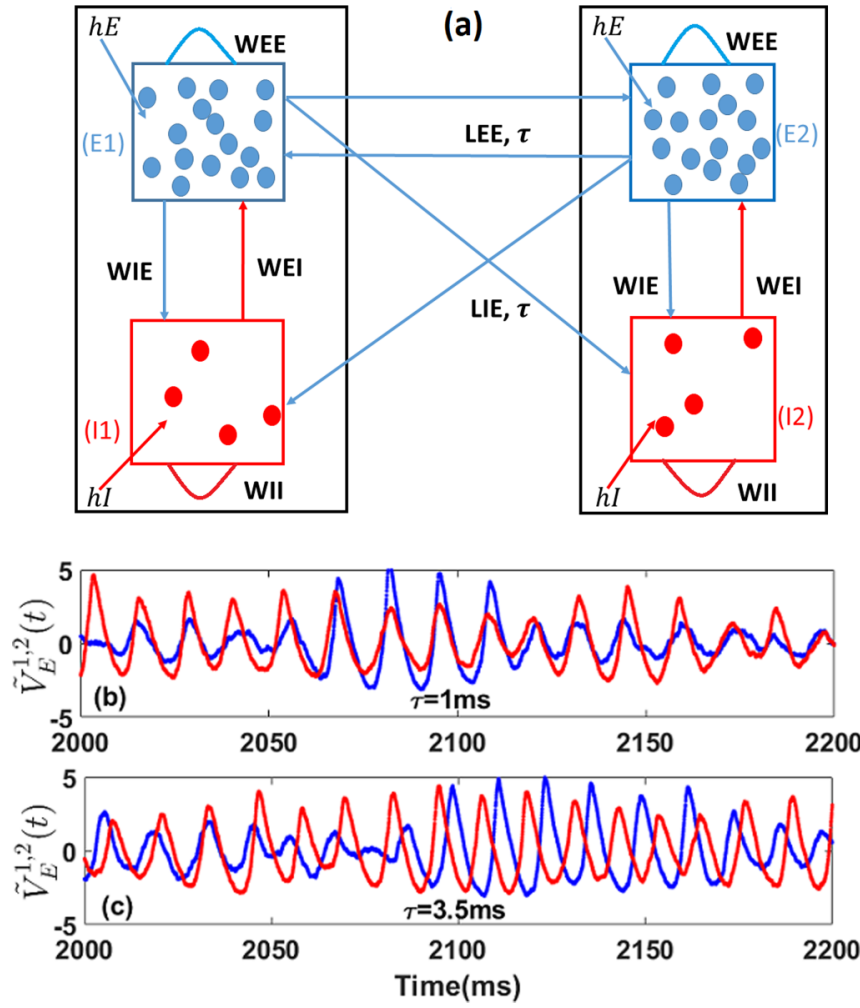

FIG. 1. Model. (a) Two delay-coupled E-I networks. (b, c) Fluctuation time series of $E_{1}$ (blue) and $E_{2}$ (red) around their equilibrium points, modeling local field potentials (LFPs), from simulations of the SWC system [Eqs. (1)] with $L_{E E}=2.0, L_{I E}=0.5$. The in-phase synchronization seen in (b) for $\tau=1 \mathrm{~ms}$ is replaced by out-of-phase behavior for $\tau=3.5 \mathrm{~ms}$ in (c). Other parameters are $W_{E E}=27.4$, $W_{I I}=1.3, W_{I E}=32, W_{E I}=26.3, h_{E}=-3.8, h_{I}=-8, \alpha_{E}=0.1$, $\alpha_{I}=0.2, \beta_{E}=1, \beta_{I}=2$, and $\sigma=0.006$. All simulations in our paper are done with the Euler-Maruyama method with time step 0.05 ms, except for Fig. 3, where the time step is shorter. A second-order Butterworth bandpass filter was applied to the fluctuations in (b) and (c) to limit frequencies to the gamma band $(30-100 \mathrm{~Hz})$.

$$
\begin{aligned}
s_{E_{i}}(t) & =W_{E E} E_{i}(t)-W_{E I} I_{i}(t)+h_{E}+L_{E E}^{i, j} E_{j}(t-\tau), \\
s_{I_{i}}(t) & =W_{I E} E_{i}(t)-W_{I I} I_{i}(t)+h_{I}^{i}+L_{I E}^{i, j} E_{j}(t-\tau),
\end{aligned}
$$

for identical and symmetrically coupled networks described in this section, $L_{E E}^{i, j}=L_{E E}, L_{I E}^{i, j}=L_{I E}$ and $h_{I}^{i}$ with $i, j=$ 1,2 and $i \neq j$. The total synaptic inputs to $\mathrm{E}$ and I populations in each network are $s_{E_{i}}(t)$ and $s_{I_{i}}(t), i=1,2$. The sigmoidal response of a neuron to its total input is $f(x)=$ $1 /(1+\exp (-x)), \xi_{E_{i}}(t)$ and $\xi_{I_{i}}(t)$ are independent Gaussian white noises, and $g_{1}$ and $g_{2}$ are population-size-dependent multiplicative noise intensities,

$$
\begin{aligned}
& g_{1}\left(E_{i}, I_{i}\right)=\sqrt{\frac{\left[1-E_{i}(t)\right] \beta_{E} f\left(s_{E_{i}}(t)\right)+\alpha_{E} E_{i}(t)}{N_{E}},} \\
& g_{2}\left(E_{i}, I_{i}\right)=\sqrt{\frac{\left[1-I_{i}(t)\right] \beta_{I} f\left(s_{I_{i}}(t)\right)+\alpha_{I} I_{i}(t)}{N_{I}}} .
\end{aligned}
$$

We define the noise intensities as

$$
\sigma_{E} \equiv\left\langle\left(g_{1}\left(E_{i}, I_{i}\right)\right)\right\rangle, \quad \sigma_{I} \equiv\left\langle\left(g_{2}\left(E_{i}, I_{i}\right)\right)\right\rangle,
$$


where \langle\rangle means the time average. Simulations reveal that the distributions of these multiplicative noise intensities are very close to Gaussians, and the values of $\sigma_{E}$ and $\sigma_{I}$ are close to the means of these distributions. We then defined the total noise as

$$
\sigma \equiv \sqrt{\sigma_{E}^{2}+\sigma_{I}^{2}}
$$

Our results below also hold for constant functions $g_{1}\left(E_{i}, I_{i}\right)=\sigma_{E}$ and $g_{2}\left(E_{i}, I_{i}\right)=\sigma_{I}$ (additive noise).

The rightmost eigenvalues of the deterministic part of Eqs. (1) are complex conjugate with negative real part $(\lambda=$ $\left.-v \pm j \omega_{0}, \quad v>0\right)$, and imaginary part in the gamma band $\left(30<\omega_{0} /(2 \pi)<100 \mathrm{~Hz}\right)$. Without noise, activities converge to a fixed point $\left(E_{10}, I_{10}, E_{20}, I_{20}\right)$. We focus on fluctuations around this point: $\tilde{V}_{E}^{i}(t)=c_{E}\left(E_{i}(t)-E_{i 0}\right)$ and $\tilde{V}_{I}^{i}(t)=$ $c_{I}\left(I_{i}(t)-I_{i 0}\right)$, where $c_{E}=\sqrt{N_{E}}$ and $c_{I}=\sqrt{N_{I}}$ for the case of the multiplicative noise and $c_{E}=c_{I}=1$ for additive noise. Time series of these local field potentials (LFPs) are illustrated in Figs. 1(b) and 1(c) with IP behavior for the smaller delay and OPL for the slightly larger one.

\section{ENVELOPE-PHASE DECOMPOSITION}

In contrast to the case $v<0$ where the limit-cycle envelopes are almost constant (in the weak noise limit), in the quasi-cycle regime the envelopes and phases (and therefore frequencies) fluctuate over a larger range. We extend a recent single quasi-cycle analysis using the SAM approximation $[19,27]$ to the coupled case of interest here, noting that the coupled system still has a deterministic fixed point. A synchronization transition study for a population of coupled quasi-cycles was recently done in this regime using a different method [28]. We write the E and I LFPs as

$$
\begin{aligned}
V_{E}^{1}(t) & =Z_{1}(t) \cos \left(\omega_{0} t+\phi_{1}(t)\right), \\
V_{I}^{1}(t) & =\alpha_{1} Z_{1}(t) \cos \left(\omega_{0} t+\phi_{1}(t)+\delta_{1}\right), \\
V_{E}^{2}(t) & =Z_{2}(t) \cos \left(\omega_{0} t+\phi_{2}(t)\right), \\
V_{I}^{2}(t) & =\alpha_{2} Z_{2}(t) \cos \left(\omega_{0} t+\phi_{2}(t)+\delta_{2}\right) .
\end{aligned}
$$

These trial solutions are motivated by our previous study [19] where the results using these solutions yielded good agreement with simulations both near and far from the Hopf bifurcation, and reproduced many features of the data. The system to which this trial solution is applied [see Appendix A, Eqs. (A2)] is also linear and stochastic, and thus harmonic solutions are a good place to start. An alternate form could be used if one wishes that a certain feature of the data needs to be reflected in the model. Inserting these expressions in the dynamics of the LFPs sustained by noise and applying the SAM we obtain the following dynamics for the amplitude and phase dynamics:

$$
\begin{aligned}
& d Z_{1}(t)=\left(-\lambda_{1} Z_{1}(t)+\frac{D_{1}}{2 Z_{1}(t)}+M_{1}^{1}\left[\phi_{1}(t)-\phi_{2}(t-\tau)\right] Z_{2}(t-\tau)\right) d t+\sqrt{D_{1}} d W_{Z_{1}}(t) \\
& d \phi_{1}(t)=\left(\Omega_{1}+M_{1}^{2}\left[\phi_{1}(t)-\phi_{2}(t-\tau)\right] \frac{Z_{2}(t-\tau)}{Z_{1}(t)}\right) d t+\frac{\sqrt{D_{1}}}{Z_{1}(t)} d W_{\phi_{1}(t)} \\
& d Z_{2}(t)=\left(-\lambda_{2} Z_{2}(t)+\frac{D_{2}}{2 Z_{2}(t)}+M_{2}^{1}\left[\phi_{2}(t)-\phi_{1}(t-\tau)\right] Z_{1}(t-\tau)\right) d t+\sqrt{D_{2}} d W_{Z_{2}}(t) \\
& d \phi_{2}(t)=\left(\Omega_{2}+M_{2}^{2}\left[\phi_{2}(t)-\phi_{1}(t-\tau)\right] \frac{Z_{1}(t-\tau)}{Z_{2}(t)}\right) d t+\frac{\sqrt{D_{2}}}{Z_{2}(t)} d W_{\phi_{2}}(t),
\end{aligned}
$$

where the functions $M_{1}^{1}, M_{1}^{2}, M_{2}^{1}$ and $M_{2}^{2}$ are given by

$$
\begin{aligned}
& M_{1}^{1}[x]=\gamma_{1}\left[\alpha_{1} C_{E E}^{12} \sin \left(x+\omega_{0} \tau+\delta_{1}\right)-C_{I E}^{12} \sin \left(x+\omega_{0} \tau\right)\right] \\
& M_{1}^{2}[x]=\gamma_{1}\left[\alpha_{1} C_{E E}^{12} \cos \left(x+\omega_{0} \tau+\delta_{1}\right)-C_{I E}^{12} \cos \left(x+\omega_{0} \tau\right)\right] \\
& M_{2}^{1}[x]=\gamma_{2}\left[\alpha_{2} C_{E E}^{21} \sin \left(x+\omega_{0} \tau+\delta_{2}\right)-C_{I E}^{21} \sin \left(x+\omega_{0} \tau\right)\right] \\
& M_{2}^{2}[x]=\gamma_{2}\left[\alpha_{2} C_{E E}^{21} \cos \left(x+\omega_{0} \tau+\delta_{2}\right)-C_{I E}^{21} \cos \left(x+\omega_{0} \tau\right)\right]
\end{aligned}
$$

with the coefficients

$$
\begin{aligned}
& \lambda_{1}=-\frac{A_{E E}^{1}+A_{I I}^{1}}{2}, \quad \lambda_{2}=-\frac{A_{E E}^{2}+A_{I I}^{2}}{2}, \\
& \gamma_{1}=\frac{1}{2 \alpha_{1} \sin \left(\delta_{1}\right)}, \quad \gamma_{2}=\frac{1}{2 \alpha_{2} \sin \left(\delta_{2}\right)},
\end{aligned}
$$

and

$$
\begin{aligned}
& D_{1}=\sqrt{\frac{\alpha_{1}^{2} \sigma_{E_{1}}^{2}+\sigma_{I_{1}}^{2}}{2\left(\alpha_{1} \sin \left(\delta_{1}\right)\right)^{2}}}, \quad D_{2}=\sqrt{\frac{\alpha_{2}^{2} \sigma_{E_{2}}^{2}+\sigma_{I_{2}}^{2}}{2\left(\alpha_{2} \sin \left(\delta_{2}\right)\right)^{2}}}, \\
& \Omega_{1}=-\omega_{0}+\gamma_{1}\left(\alpha_{1} \cos \left(\delta_{1}\right)\left(A_{E E}^{1}-A_{I I}^{1}\right)+\alpha_{1}^{2} A_{E I}^{1}-A_{I E}^{1}\right) \text {, } \\
& \Omega_{2}=-\omega_{0}+\gamma_{2}\left(\alpha_{2} \cos \left(\delta_{2}\right)\left(A_{E E}^{2}-A_{I I}^{2}\right)+\alpha_{2}^{2} A_{E I}^{2}-A_{I E}^{2}\right) \text {. }
\end{aligned}
$$

Here $d W_{k}, k=Z_{1,2}, \phi_{1,2}$, are independent Brownian motions, and the parameters $\lambda_{1,2}, \Omega_{1,2}, \delta_{1,2}, \alpha_{1,2}, C_{E E}^{12}, C_{E E}^{21}$, and $C_{I E}^{12}$, $C_{I E}^{21}$ depend on the network parameters described above (see Appendix A). Specifically, $\alpha_{1,2}$ and $\delta_{1,2}$ are the amplitude ratio and phase difference between the I and $\mathrm{E}$ populations in networks 1 and 2, respectively.

The dependencies of the parameters of the envelope-phase equations on those in the original SWC system are not trivial, as their definitions that follow Eqs. (A2) in the full derivation in Appendix A reveal. And a number of parameters depend 

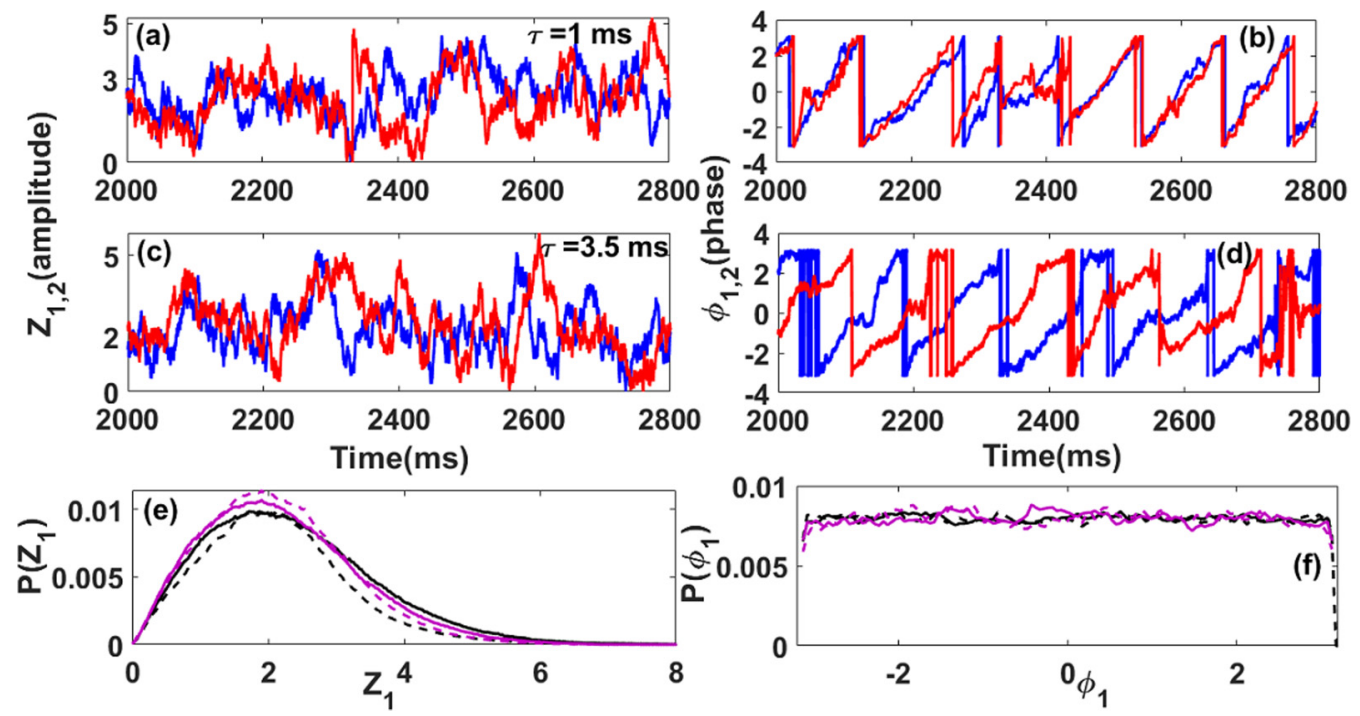

FIG. 2. Coupled envelope and phase dynamics. Envelope time series for $E_{1}$ (blue) and $E_{2}$ (red) populations for coupling $L_{E E}=2.0$, $L_{I E}=0.5$ and delay (a) $\tau=1 \mathrm{~ms}$ and (c) $3.5 \mathrm{~ms}$. Corresponding phase dynamics are shown in (b) and (d). In-phase locking is seen for $\tau=1$ $\mathrm{ms}$, and out-of-phase locking for $\tau=3.5 \mathrm{~ms}$ as in Figs. 1(b) and 1(c). (e) Envelope and (f) phase probability distributions from simulations of the SWC model [Eqs. (1), solid lines] through the Hilbert transform and the SAM theory [Eqs. (7), dashed lines], for $\tau=1 \mathrm{~ms}$ (magenta) and $\tau=3.5 \mathrm{~ms}$ (black). Parameters are as in Fig. 1 .

on the fixed point, which is a function of all the parameters. For the symmetric case, we have $\alpha_{1}=\alpha_{2}=\alpha, \delta_{1}=\delta_{2}=\delta$, $\lambda_{1}=\lambda_{2}, \Omega_{1}=\Omega_{2}=\Omega_{0}, D_{1}=D_{2}=D, C_{E E}^{12}=C_{E E}^{21}=C_{E E}$, and $C_{I E}^{12}=C_{I E}^{21}=C_{I E}$. The envelope-phase dynamics therefore simplify to

$$
\begin{aligned}
\frac{d Z_{1}}{d t} & =-\lambda_{1} Z_{1}+\frac{D}{2 Z_{1}}+M_{1}\left[\phi_{1}-\phi_{2 \tau}\right] Z_{2 \tau}+\sqrt{D} \xi_{Z_{1}} \\
\frac{d \phi_{1}}{d t} & =\Omega_{0}+M_{2}\left[\phi_{1}-\phi_{2 \tau}\right] \frac{Z_{2 \tau}}{Z_{1}}+\frac{\sqrt{D}}{Z_{1}} \xi_{\phi_{1}}, \\
\frac{d Z_{2}}{d t} & =-\lambda_{1} Z_{2}+\frac{D}{2 Z_{2}}+M_{1}\left[\phi_{2}-\phi_{1 \tau}\right] Z_{1 \tau}+\sqrt{D} \xi_{Z_{2}}, \\
\frac{d \phi_{2}}{d t} & =\Omega_{0}+M_{2}\left[\phi_{2}-\phi_{1 \tau}\right] \frac{Z_{1 \tau}}{Z_{2}}+\frac{\sqrt{D}}{Z_{2}} \xi_{\phi_{2}},
\end{aligned}
$$

where $\phi_{i \tau} \equiv \phi_{i}(t-\tau), Z_{i \tau} \equiv Z_{i}(t-\tau)$, and

$M_{1}=\frac{1}{2 \alpha \sin (\delta)}\left[\alpha C_{E E} \sin \left(x+\omega_{0} \tau+\delta\right)-C_{I E} \sin \left(x+\omega_{0} \tau\right)\right]$,

$M_{2}=\frac{1}{2 \alpha \sin (\delta)}\left[\alpha C_{E E} \cos \left(x+\omega_{0} \tau+\delta\right)-C_{I E} \cos \left(x+\omega_{0} \tau\right)\right]$.

Here again, $\lambda_{1}, D, \Omega_{0}, C_{E E}$, and $C_{I E}$ depend on network parameters and are the same for both networks, but $\xi_{k}, k=$ $\left\{Z_{1,2}, \phi_{1,2}\right\}$, are again independent Gaussian white noises as in the general case above.

The E envelopes and phases from Eqs. (7) are shown in Figs. 2(a)-2(d). In Figs. 2(e) and 2(f), good agreement is found between probability distributions of the envelopes and phases from simulations of the SAM Eqs. (7) and of the SWC Eqs. (1). Agreement improves for weaker noise and closer proximity to the Hopf bifurcation. The phase dynamics $\phi_{i}(t)$ here differs from that of the usual Hilbert transform by the deterministic rotation $\omega_{0} t$. The phase difference $\Delta \phi(t)=$ $\phi_{1}(t)-\phi_{2}(t)$ describes the same quantity as the difference of the phases extracted using the Hilbert transform.

\section{QUASI-CYCLE PHASE SYNCHRONIZATION}

We first derive phase-locking dynamics from the envelopephase system in Eqs. (7) using $\Delta \phi(t) \equiv \phi_{1}(t)-\phi_{2}(t), \theta(t) \equiv$ $\phi_{1}(t)+\phi_{2}(t), X \equiv(\theta(t)-\theta(t-\tau)) / 2$, and $Y \equiv(\Delta \phi(t)+$ $\Delta \phi(t-\tau)) / 2$ :

$$
\begin{aligned}
d \Delta \phi(t) & =-2 M_{1}[X(t)] \sin [Y(t)] d t+\frac{\sqrt{D}}{Z_{1}(t)} d W_{1}(t), \\
d \theta(t) & =2\left(\Omega_{0}+M_{2}[X(t)] \cos [Y(t)]\right) d t+\frac{\sqrt{D}}{Z_{1}(t)} d W_{2}(t),
\end{aligned}
$$

where we assumed $Z_{1} \sim Z_{2}, W_{1}=W_{\phi_{1}}-W_{\phi_{2}}$, and $W_{2}=$ $W_{\phi_{1}}+W_{\phi_{2}}$. Following Ref. [29], we seek the deterministic solutions of Eqs. (8) in the form $(\Delta \phi(t), \theta(t))=\left(\Delta \phi^{*}, \Omega t\right)$. Stability is governed by

$$
F\left(\Delta \phi^{*}\right)=-2 M_{1}[\Omega \tau / 2] \sin \left(\Delta \phi^{*}\right),
$$

where $\Omega$ is a solution to $\Omega=2\left(\Omega_{0}+M_{2}\left[\frac{\Omega \tau}{2}\right] \cos \left(\Delta \phi^{*}\right)\right)$, $C_{I E}, C_{E E}, \omega_{0}, \tau \geqslant 0$ and $\delta \leqslant 0$. Either IP or AP stable solutions exist, depending on $C_{I E}, C_{E E}, \omega_{0}, \tau$, and $\delta$. The transition between IP and AP occurs at a critical delay $\tau^{*}=$ $\left(2 /\left(2 \omega_{0}+\Omega\right)\right) \operatorname{atan}\left[\left(\frac{-\alpha \sin (\delta) C_{E E}}{\alpha C_{E E} \cos (\delta)-C_{I E}}\right)\right]$. This implies the necessary condition $C_{E E}>C_{I E} /(\alpha \cos (\delta))$ for the existence of $\mathrm{AP}$ solutions. For $\tau=0$, the deterministic stability function is reduced to $F\left(\Delta \phi^{*}\right)=-\left[C_{E E}\right] \sin \left[\Delta \phi^{*}\right]$. The only stable solutions correspond to IP. The noise-free quasi-cycle phase dynamics converge to a fixed point, and thus do not allow spontaneous symmetry breaking. SAM theory leads to the stochastic stability function

$$
\tilde{F}(\Delta \phi)=M_{2}\left[\phi_{1}-\phi_{2 \tau}\right] \frac{Z_{2 \tau}}{Z_{1}}-M_{2}\left[\phi_{2}-\phi_{1 \tau}\right] \frac{Z_{1 \tau}}{Z_{2}},
$$



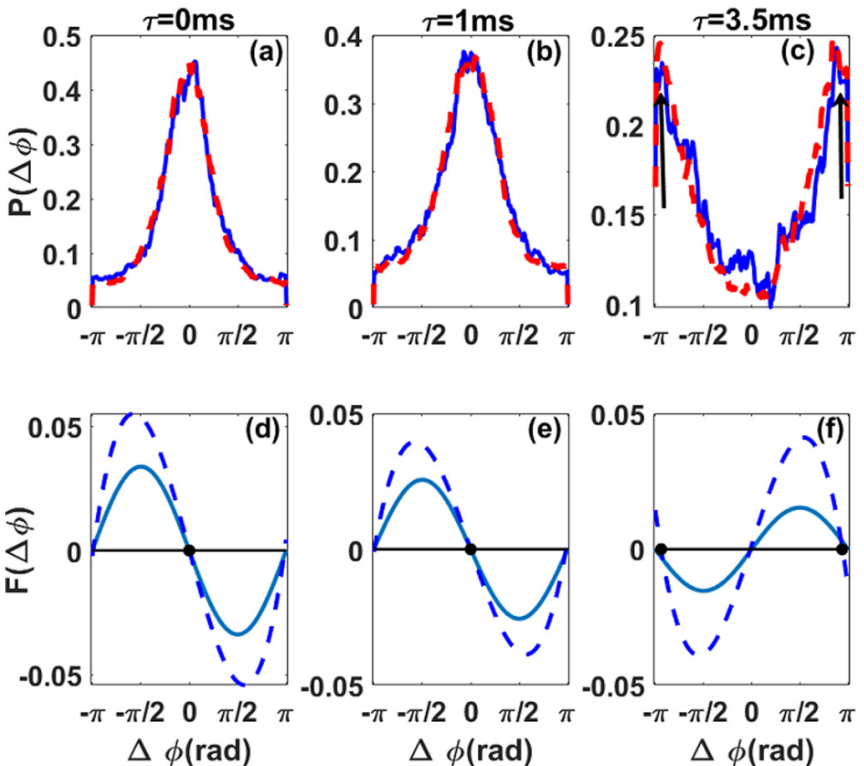

FIG. 3. OPL for quasi-cycles. (a-c) Phase difference probability distributions for $\tau=0,1$, and $3.5 \mathrm{~ms}$ from SAM theory (solid blue) and Hilbert transforms of simulations of SWC Eqs. (1) (dashed red lines). For small $\tau$ (a,b), densities peak at $\Delta \phi=0$ (IP), while for larger $\tau$ (c) OPL is seen with two symmetric peaks away from zero (IP) and $\pm \pi$ (AP) (see vertical black arrows). Good agreement is seen in all cases. (d-f) Stability functions for, respectively, $(\mathrm{a}-\mathrm{c})$. Deterministic stability functions, Eq. (9) (solid blue), with stable fixed points (black dots) located at zero for IP (d, e) and at $\pm \pi$ for AP (f). The stochastic stability function, Eq. (10) (dashed blue), evaluated from simulations with noise, followed by smoothing with a third degree polynomial, show the same fixed points for $(d, e)$, but OPL instead of AP for (f) as in (c). Parameters are as in Fig. 1, except that the time step has been reduced to $0.01 \mathrm{~ms}$ for both the SAM and SWC simulations.

which can be evaluated from simulations. Figures 3(d) and 3(e) reveal that for weak delay, the stochastic and deterministic stability functions have the same stable fixed points, which correspond to IP states. But for the larger delay in Fig. 3(f), $\tilde{F}$ exhibits two symmetric stable fixed points at locations $\Delta \phi^{*}=-\beta, \beta$ with $0<|\beta|<\pi$, corresponding to OPL states. Thus, for quasi-cycles, OPL relies on both delay and noise.

\section{DELAY AND NOISE INDUCE OUT-OF-PHASE LOCKING}

\section{A. Out-of-phase locking in E-I networks}

\section{Limit-cycle regime}

The delay and noise intensity are now varied to reveal their effect on phase locking. We first confirm previously published findings that OPL occurs via SSB in the limit-cycle regime $[13,14]$ in Fig. 4(a). This is done by looking at the deterministic situation where each network is in the limit-cycle regime prior to its coupling to the other network. The coupling induces OPL at a critical value of the delay $\tau_{\text {crit }} \approx 1.6 \mathrm{~ms}$ [Fig. 4(a), black dots]. One network becomes the leader and the other the laggard; the identity of each one depends on the initial conditions. There is no need for noise to observe such
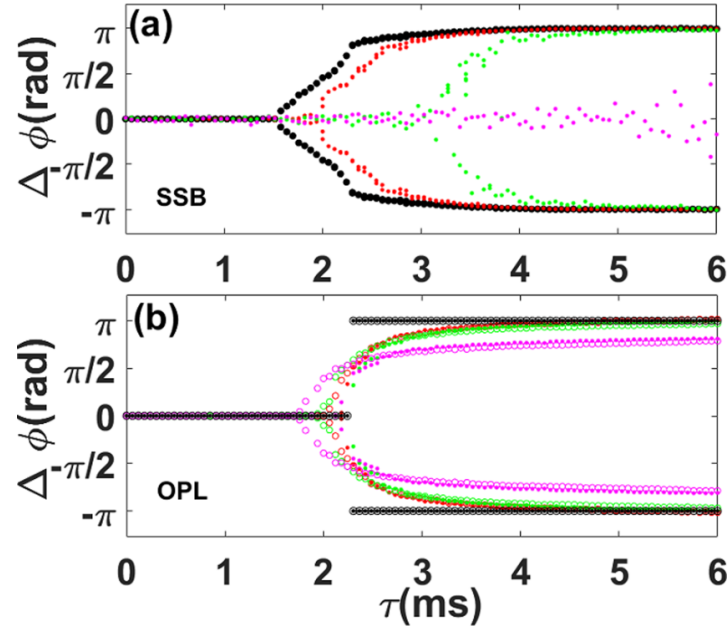

FIG. 4. Noise and delay-induced OPL. (a) Coupled limit cycles. In the deterministic case ( $\sigma=\sqrt{\sigma_{E}^{2}+\sigma_{I}^{2}}=0$, solid black dots), a bifurcation occurs from IP to OPL via spontaneous symmetry breaking at a critical delay value. OPL is further delayed (and IP stabilized) as $\sigma$ increases (red, 0.0006; green, 0.0018; magenta, 0.0156). (b) Coupled quasi-cycles. Only a bifurcation from IP to AP is seen for $\sigma=0$ (black). OPL is observed only in the noisy case. Phase differences are computed by applying Hilbert transforms to simulations of the SWC system [Eqs. (1)] [solid dots in (a) and (b), except for the black dots in (b)] and from the solutions of the SAM theory [open circles in (b)]. Parameters for (a) are $L_{E E}=1, L_{I E}=0.5$, and $W_{E E}=30.4$. In (b), we have $L_{E E}=2, L_{I E}=0.5$, and $W_{E E}=27.4$. Other parameters are as in Fig. 1. Due to the transient synchrony nature of the oscillations, phase differences were computed using the correlation function in (b) and other bifurcation diagrams below.

OPL states; i.e., OPL is a deterministic nonlinear phenomenon $[13,14]$.

However, there is no reversal of leadership unless there is an external intervention to induce it $[14,15]$. Such lack of leadership exchange is thought to be problematic for interareal brain communication, since information is preferentially shared when one network leads the other, and dynamic exchange of leadership is required for an efficient bidirectional communication between areas [8]. The main effect of noise on the deterministic SSB property is to postpone the transition between the IP and OPL regimes, in proportion to the noise intensity, as well as induce changes in leadership. The initial condition will bias the solution towards one leader. At low noise, leadership changes can occur but are increasingly rare as $\tau$ moves further beyond the deterministic bifurcation point $\tau_{\text {crit }}$.

\section{Quasi-cycle regime}

The underlying deterministic dynamics of the quasi-cycles decay to the fixed point in an underdamped manner, but a phase relationship between the two networks can nevertheless be computed during such decays. Then, only IP and AP are seen, with a transition from IP to AP at a critical delay $\tau=\tau^{*}$. This is shown in Fig. 4(b) using linear stability analysis of the SWC system (solid black dots), as well as the SAM-derived stability function $F\left(\Delta \phi^{*}\right)$ in Eq. (9) (black circles). Noise then shifts the trivial IP and AP locations and induces OPL. 
The noise allows the dynamics to sample both the IP and the neighboring AP attractors, yielding on average two OPL states. At $\tau^{*}$, the noise samples each attractor similarly, with OPL around $\pm \pi / 2$. Below $\tau^{*}$, the IP attractor in the full SWC simulations appears destabilized by noise, but it is difficult to resolve whether this also represents an advancement of the bifurcation. This is due to critical slowing down near the bifurcation, which makes the simulations converge prohibitively slowly to pinpoint the bifurcation exactly in the presence of noise. But clearly the situation differs qualitatively from the postponement observed in Fig. 4(a) for the limit cycles.

Beyond $\tau^{*}$, the noise increasingly samples the AP attractor. The OPL asymptotically tends to the deterministic AP value $\Delta \phi^{*}=|\pi|$ in a time proportional to noise strength. The abrupt IP-AP transition observed in the transient deterministic dynamics is smoothed by the noise and arises for smaller delays. It is important to mention that when the delay is varied in Fig. 4, the intrinsic frequency of the networks remains almost constant. The main effect of the propagation delay is to induce AP states which are smoothed by noise to give rise to OPL states.

Such OPL attractors reflect a noise-induced transition where the parameter range for OPL increases with noise intensity. The networks also exchange leadership over time due to the noise, without external pulses, yielding a "dynamic OPL" for quasi-cycles. This suggests that coupled quasi-cycles are good candidates for flexible inter-areal network communication. OPL states are also excitation dependent, since the aforementioned necessary condition implies that strong $L_{E E}$ and weak $L_{I E}$ values promote OPL.

Additive rather than multiplicative noises in the model formulation lead to similar results (not shown), as can be verified by replacing $g_{1}\left(E_{i}, I_{i}\right)$ and $g_{2}\left(E_{i}, I_{i}\right)$ by their respective numerically determined means $\sigma_{E}$ and $\sigma_{I}$ (these densities are approximately Gaussian).

The mechanism of OPL in the coupled quasi-cycle regime is thus different from the one in the limit-cycle regime. The presence of noise is critical for OPL. The noise also allows a continual random exchange of leadership between the two networks; in fact, since the noise is required for sustained quasi-cycle behavior, OPL is always present. As in the coupled limit-cycle case, the initial condition will bias the leadership towards one network, and subsequent leadership exchanges are increasingly rare as the delay moves beyond the bifurcation point, or when the noise intensity is small. But in principle, each network spends half the time being the leader, as could be verified with long simulations (and perhaps unreasonably long when the noise is small or the delay is large). This has already been observed in another purely computational study [8].

Figure 4(b) also shows that the SAM theory predicts OPL states in the quasi-cycle regime. Further, it shows that the bifurcation is advanced to smaller and smaller delay values the stronger the noise is; this contrasts with what is seen in the coupled limit-cycle case. More extensive simulations of the full network SWC equations are needed [i.e., more closed symbols closer to the zero axis need to be computed from very long simulations in Fig. 4(b)] to determine whether advancement occurs, and if so, how well it agrees with the SAM theory. The advancement here also contrasts with other
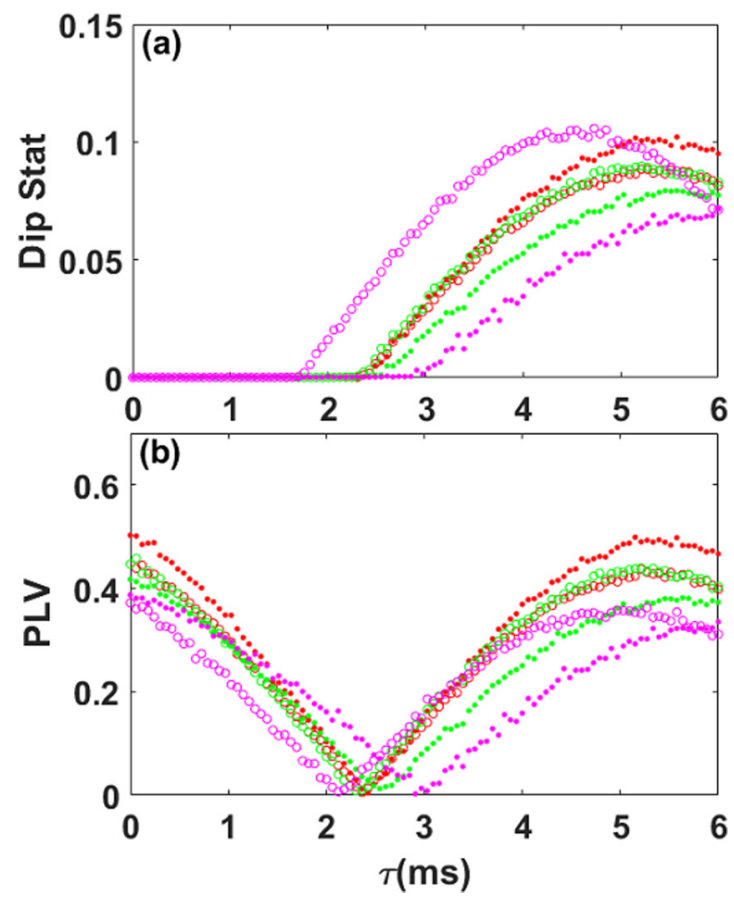

FIG. 5. Dip statistic and phase-locking value show signatures of OPL. (a) Dip statistic versus lag assesses the presence of bimodality in the density of the time series, which is in agreement with the appearance of OPL in Figs. 3 and 4. (b) Phase-locking value versus lag measures the degree of phase synchronization between two time series. The PLV was computed from the phase time series $\phi_{1}(t)$ and $\phi_{2}(t)$ using both the Hilbert transform (solid dots) and the SAM (circles). The PLV values are weaker at the transition between IP and AP locking, revealing the appearance of OPL states. All the parameters are the same as in Fig. 4(b).

delay-differential equations where additive or multiplicative noise postpones a Hopf bifurcation [30].

The dip test for bimodality was performed on the $\Delta \phi(t)=$ $\phi_{1}(t)-\phi_{2}(t)$ time series [Fig. 5(a)]. It is a statistical test that measures the level of multimodality of the density of a given time series (see Appendix D). A value near zero indicates a unimodal time series, while increasing values of the dip statistic are associated with more than one mode in the density. We observe an increase of the dip values close to the deterministic transition between IP and AP locking. This shows the presence of multimodality (bimodality in this specific case) in the distribution of phase-difference values in the time series, and therefore the presence of OPL. In fact, it exposes a transition from zero to nonzero dip values very near the same points as in Fig. 4, as well as in Fig. 3(c) with modes located at $0<|\beta|<|\pi|$. The dip statistic thus corroborates the conclusion of bimodality, and therefore OPL. We also computed the phase-locking value (PLV) to measure the synchronization strength [12] (see Appendix D). Higher values of the PLV correspond to strong synchronization. The PLV is seen to capture well the transition between IP and OPL. PLV analysis reveals that the synchronization strength increases away from the IP-AP bifurcation in either direction [Fig. 5(b)]. The PLV is nonzero, and shows a minimum near the same transition points as in Fig. 4(b). We also observe a 
discrepancy between the SAM and the Hilbert transform at large noise intensity for both the dip value and the PLV.

We finally note that the coupling delay has little effect on the oscillation frequency of each network. The frequency of the coupled network is mainly determined by the parameters of each network and the connection coefficients.

\section{B. Effect of asymmetric coupling and network heterogeneity}

The case we have considered so far involves two identical networks that are coupled reciprocally using the same delays and coupling strengths. This is an oversimplification, as real neural networks are never identical and the connectivity is rarely symmetric. Is the mechanism of delay and noise-induced OPL still present when symmetry and homogeneity conditions are not present? Much work is required to fully address these issues. Our immediate, more restricted goal here is to show a generic effect of relaxing the requirement of symmetric coupling or network homogeneity. Specifically, we first consider the case where identical networks are asymmetrically coupled. Afterwards, we look at the heterogeneous case when nonidentical networks are symmetrically coupled.

The asymmetric case is set up by slightly decreasing the excitatory coupling from the first to the second network $\left(L_{E E}^{21}=\right.$ $1.5, L_{E E}^{12}=2$ ). Figure 6(a) shows that the asymmetry induces a small phase difference between the two networks even for the smallest delays, leading to a dominant directionality. Nevertheless, the mechanism of delay- and noise-induced OPL is still present, although with an intrinsic bias compared to the symmetric case. When the delay increases, a second branch eventually appears; i.e., another OPL state is induced. As in all the bifurcation diagrams up to now for stochastic dynamics, we plotted only the local extrema of the phase difference distribution. For a given delay, the two states are not symmetric as in the former case, but rather one state is more stable than the other.

In the heterogeneous case in Fig. 6(b), the external inhibitory input to the second network is slightly increased, but the connectivity coefficients are symmetric, and all other parameters identical. As in the asymmetric case, this also induces a phase difference between the networks. The mechanism of noise- and delay-induced OPL is still present, but again with a bias. The heterogeneous case is qualitatively similar to the asymmetric case. All branches increase (decrease) towards $\pm \pi$ when the delay is large.

Hence, for both of these specific examples of asymmetry and heterogeneity, the mechanism of delay- and noise-induced OPL occurs, along with dynamical changes in leadership. However, the amount of time one network leads the other is not the same as in the homogeneous and symmetric case. In fact one network leads the other one most of the time depending on the phase relation induced by the asymmetry or the heterogeneity. This mechanism is observed and persists when the asymmetry is weak and the heterogeneous networks are nearly identical. If the asymmetry is strong or the two networks are too different, the phase relation will be imposed by such asymmetry or heterogeneity. Then, one network will almost always be the leader, as the noise will rarely induce switches to the less stable state. Dynamic OPL is revealed
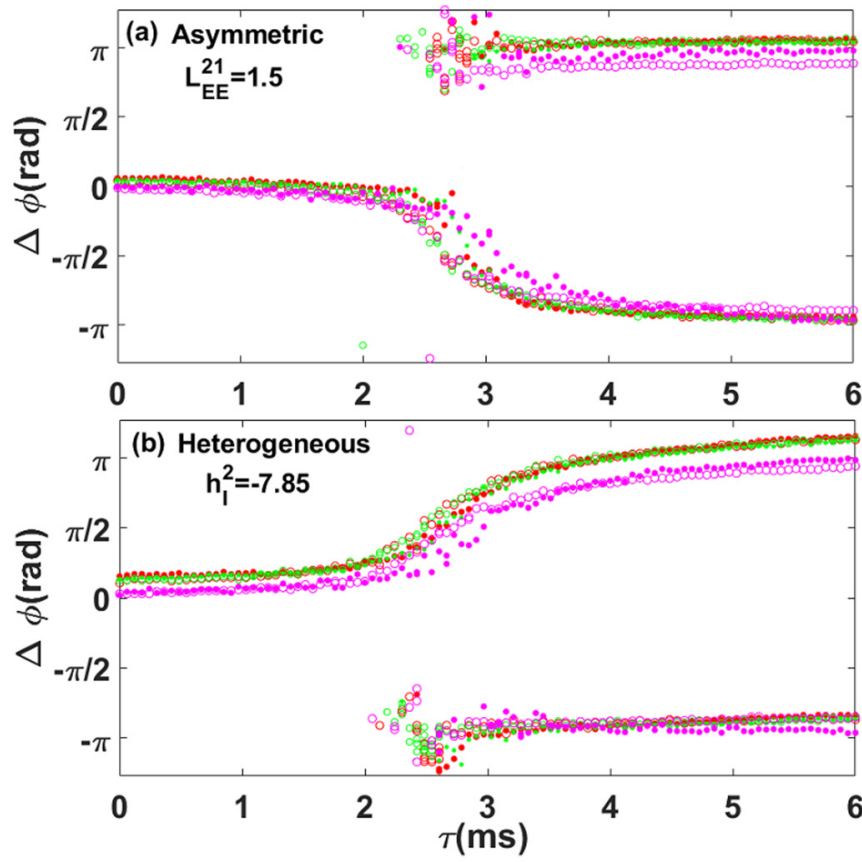

FIG. 6. Noise- and delay-induced OPL in homogeneous asymmetrically coupled or heterogeneous but symmetrically coupled E-I networks. (a) The asymmetric case where the excitatory connectivity from the first to second network is slightly decreased compared to the reverse excitatory connectivity $\left(L_{E E}^{21}=1.5, L_{E E}^{12}=2\right)$. The other parameters are identical for the two networks. (b) The heterogeneous case where the inhibitory input to the second network is slightly increased compared to the same input applied in the first network $\left(h_{I}^{1}=\right.$ $\left.-8, h_{I}^{2}=-7.85\right)$. Connectivities are kept symmetric as previously in Fig. 4. For all panels and figures, solid dots are phase-locking states obtained using the Hilbert transform, while circles are obtained through the SAM [Eqs. (5)]. Red corresponds to weak, green to intermediate, and magenta to strong values of the noise intensity (values are as in Fig. 4).

using numerical simulations of Eqs. (1) followed by Hilbert transforms, as well as the SAM in Eqs. (5) (for the heterogeneous and asymmetric case) and Eqs. (7) (for the symmetric and homogeneous case).

Results from the Hilbert transform and the SAM are in good agreement for weak and intermediate values of the noise intensity (see red and green solid dots and circles in Fig. 6). From the large noise intensity, the results for the SAM and the Hilbert transform start to diverge as observed in the magenta solid dots and circles in Fig. 6. The dynamics derived from the SAM [Eqs. (5) and (7)] provide a good approximation for the envelope and phase of the LFPs derived in Eqs. (1) for weak and intermediate values of the noise for the E-I network. However, quasi-cycles are also present in purely inhibitory networks. A natural question will be to understand the phase-locking mechanism of such quasi-cycles.

\section{OPL in inhibitory networks}

We finally considered two coupled identical inhibitory (I-I) networks. Each isolated I-I population with its all-to-all delayed inhibition can also exhibit either a noisy limit cycle or quasi-cycle $[9,10,31,32]$. We coupled the two I-I populations with delayed long-range excitatory connections in the quasi- 

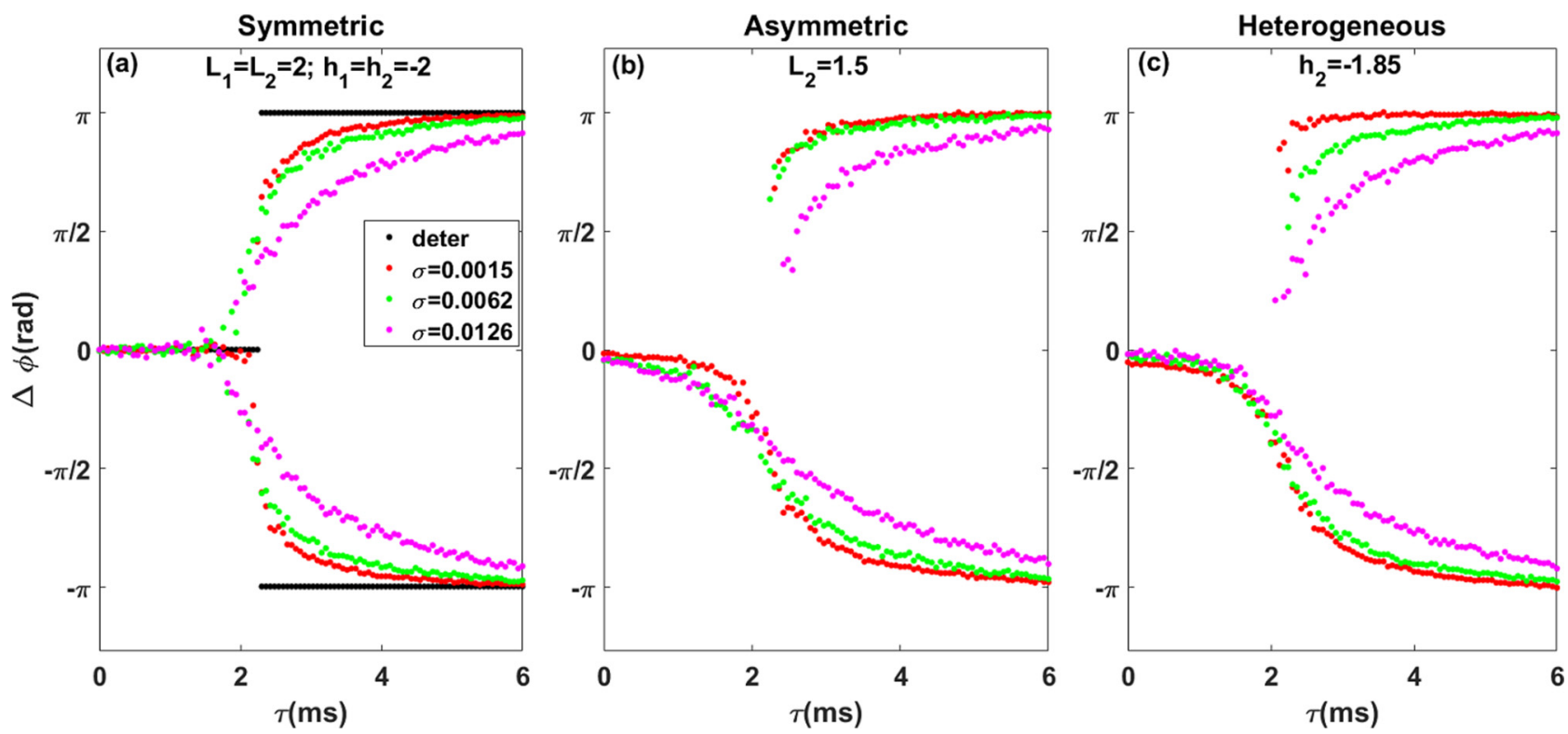

FIG. 7. Noise-induced out-of-phase locking in two purely inhibitory networks coupled via long-range delayed excitatory connections (ING mechanism). For all curves, red corresponds to weak, green to intermediate, and magenta to strong noise. The respective values of the noise intensities are specified in the legend of the left panel. (a) The symmetric case with black dots corresponds to the deterministic phase locking computed through linear stability analysis. (b) The asymmetric case where the connectivity is enhanced in one direction compared to the other. (c) The heterogeneous case where the external current applied to the second network is slightly greater. We observe that noise and delay induce out-of-phase locking; phase locking persists for the asymmetric and heterogeneous cases. Simulations are performed using the phase signal extracted through the Hilbert transform. For the symmetric case, the parameters are chosen as follows: $\alpha_{I}=0.1, \beta_{I}=2, W=30, \hat{\tau}=5.5$ $\mathrm{ms}, h_{1}=h_{2}=-2$, and $L_{1}=L_{2}=2$. For the asymmetric case, we have slightly decreased $L_{2}$ to 1.5 and for the heterogeneous case we have increased $h_{2}$ to -7.85 . The noise intensities are $\sigma \approx \sigma_{1} \approx \sigma_{2}$.

cycle regime as for the E-I networks discussed until now. The dynamics of the model is given by

$$
\begin{aligned}
& \frac{d I_{1}(t)}{d t}=-\alpha_{I} I_{1}(t)+\left[1-I_{1}(t)\right] \beta_{I} f\left(s_{I_{1}}(t)\right)+g_{1}\left(I_{1}\right) \xi_{I_{1}}(t), \\
& \frac{d I_{2}(t)}{d t}=-\alpha_{I} I_{2}(t)+\left[1-I_{2}(t)\right] \beta_{I} f\left(s_{I_{2}}(t)\right)+g_{2}\left(I_{2}\right) \xi_{I_{2}}(t), \\
& s_{I_{1}}(t)=h_{1}-W I_{1}(t-\hat{\tau})+L_{1} I_{2}(t-\tau), \\
& s_{I_{2}}(t)=h_{2}-W I_{2}(t-\hat{\tau})+L_{2} I_{1}(t-\tau), \\
& g_{1}\left(I_{1}\right)=\sqrt{\frac{\alpha_{I} I_{1}(t)+\left[1-I_{1}(t)\right] \beta_{I} f\left(s_{I_{1}}(t)\right)}{N}} \\
& g_{2}\left(I_{2}\right)=\sqrt{\frac{\alpha_{I} I_{2}(t)+\left[1-I_{2}(t)\right] \beta_{I} f\left(s_{I_{2}}(t)\right)}{N}}
\end{aligned}
$$

As in the case of the coupled E-I networks, we define the noise intensities for each network as

$$
\sigma_{1}=\left\langle\left(g_{1}\left(I_{1}\right)\right)\right\rangle \text { and } \sigma_{2}=\left\langle\left(g_{2}\left(I_{2}\right)\right)\right\rangle .
$$

For this case, an envelope-phase decomposition using the SAM as in the case of the coupled E-I networks is not yet available. We thus only consider numerical simulations and linear stability analysis. We define the LFPs as $\tilde{V}_{I_{1}}(t)=$ $c\left(I_{1}(t)-I_{10}\right)$ and $\tilde{V}_{I_{2}}(t)=c\left(I_{2}(t)-I_{20}\right)$, where $I_{10}$ and $I_{20}$ are the stable fixed points of networks 1 and 2, $c=\sqrt{N}$ for the case of the multiplicative noise, and $c=1$ for the additive noise. The phase difference is again $\Delta \phi(t)=\phi_{1}(t)-\phi_{2}(t)$, where $\phi_{1}(t)$ is the phase of the signal $\tilde{V}_{I_{1}}(t)$ and $\phi_{2}(t)$ the one of $\tilde{V}_{I_{2}}(t)$, both extracted through the Hilbert transform. We perform the same analysis as in the case of the coupled E-I networks.

We first consider the symmetric case, choosing the delay as the bifurcation parameter for different noise intensities $\sigma$. We observe in Fig. 7 that the mechanism of delay and noiseinduced OPL is qualitatively the same as that observed in the case of coupled E-I networks. The noise smoothes the abrupt transition between the deterministic IP and AP locking states, and induces OPL states. We also consider the symmetric and heterogeneous cases as previously described for the coupled E-I networks. The mechanism of delay- and noise-induced OPL again survives these losses of symmetry, but with a bias compared to the symmetric case. The presence of this mechanism in the case of purely inhibitory networks suggests that it only depends on the dynamical regime of the oscillations in each network, i.e., on their noise-induced nature. We then expect to observe similar delay- and noise-induced OPL more generally if the corresponding networks in isolation exhibit quasi-cycles.

\section{DISCUSSION}

In summary, a robust noise-induced, delay-dependent OPL mechanism was revealed by an envelope-phase decomposition of the coupled quasi-cycle dynamics. Although no spontaneous symmetry breaking arises, the noise samples attractor dynamics corresponding to both IP and AP dynamics, leading to OPL well before the deterministic bifurcation point. The lead-lag relationship displays random reversals, an intrinsic 
property of the coupled system. This mechanism is observed in both coupled PING- and ING-type systems. It persists but with a bias when the networks are weakly asymmetrically coupled, or when they are heterogeneous but nearly identical.

The SAM used here requires underdamped oscillatory motion in each network prior to coupling. It has been extended here to include delays. It allows an accurate description of the envelope and phase dynamics of the nonlinear PING LFPs for weak and intermediate value of the noise. It constitutes an appropriate theoretical framework for the study of phase synchronization in quasi-cycles. However, we found that for strong values of the noise intensity, the agreement with the Hilbert transform was not so good. There may be many reasons for discrepancy. First, we compare the SAM equations which are derived from the linear equations sustained by noise [see Appendix A, Eqs. (A2)] with the full SWC which is a stochastic nonlinear model. Neglecting nonlinear terms to calculate the SAM could lead to some discrepancy. In fact, for high values of the noise intensity, the agreement between the network frequencies from the full nonlinear SWC model and the linear noise approximation (LNA) dynamics significantly worsens (not shown). Since the phase relation depends on the network frequencies, the immediate consequence is a disagreement between the SAM and the full nonlinear SWC model.

Second, we found that numerical simulations of the envelope-phase dynamics obtained via the SAM can be problematic. The classical Euler-Maruyama iterative scheme was inaccurate in the sense that the envelope process could be negative during the simulation process. To deal with this pathological issue, we consider a very small time step, and replace the envelope processes by their absolute values at each simulation time step. This allows the processes to remain positive. This numerical scheme may cause convergence inaccuracies when the amplitude process is close to zero, particularly for strong noise.

Despite these limitations, the SAM method remains a good theoretical framework for the study of envelope and phase dynamics of isolated and coupled quasi-cycles. It exhibits all the qualitative behavior of phase and amplitude dynamics present in the full nonlinear SWC model. The mechanism of delay-dependent, noise-induced out-of-phase locking exposed here through the SAM and the Hilbert transform of Eqs. (1) is robust, flexible, and general for rhythm-based communication purposes. The potential ability to communicate under the realistic conditions reported here opens the way for information transfer studies to obtain deeper insights into activity coordination in complex networks. This would require extending the methods used here to dimensions greater than two and with the necessary network topology. Also, the inclusion of more realistic external signals, such as correlated noise or periodic inputs, promises to yield interesting phenomena.

\section{ACKNOWLEDGMENT}

This work was supported by NSERC Canada.

\section{APPENDIX A: LINEAR NOISE APPROXIMATION}

We first consider the deterministic part of Eqs. (1) $\left(g_{i}=0\right.$, $i=1,2)$. Their corresponding fixed points $\left(E_{10}, I_{10}, E_{20}, I_{20}\right)$ can be obtained as the solutions of the following system:

$$
\begin{aligned}
-\alpha_{E} E_{10}+\left(1-E_{10}\right) \beta_{E} f\left(s_{E_{10}}\right) & =0, \\
-\alpha_{I} I_{10}+\left(1-I_{10}\right) \beta_{I} f\left(s_{I_{10}}\right) & =0, \\
-\alpha_{E} E_{20}+\left(1-E_{20}\right) \beta_{E} f\left(s_{E_{20}}\right) & =0, \\
-\alpha_{I} I_{20}+\left(1-I_{20}\right) \beta_{I} f\left(s_{I_{20}}\right) & =0,
\end{aligned}
$$

with

$$
\begin{aligned}
s_{E_{10}} & =W_{E E} E_{10}-W_{E I} I_{10}+h_{E}+L_{E E}^{12} E_{20}, \\
s_{I_{10}} & =W_{I E} E_{10}-W_{I I} I_{10}+h_{I}^{1}+L_{I E}^{12} E_{20}, \\
s_{E_{20}} & =W_{E E} E_{20}-W_{E I} I_{20}+h_{E}+L_{E E}^{21} E_{10}, \\
s_{I_{20}} & =W_{I E} E_{20}-W_{I I} I_{20}+h_{I}^{2}+L_{I E}^{21} E_{10} .
\end{aligned}
$$

We are interested in the parameter regime where such a fixed point is a stable focus. The dynamics therefore converge in a decaying oscillatory manner toward the fixed point in the absence of noise. When noise is added, the dynamics are fluctuations around the fixed points. Following the system size expansion [33-36], these fluctuations can be sought in the following form:

$$
\tilde{V}_{E}^{i}=\sqrt{N_{E}}\left(E_{i}-E_{i 0}\right), \quad \tilde{V}_{I}^{i}=\sqrt{N_{I}}\left(I_{i}-I_{i 0}\right), \quad i=1,2 .
$$

Plugging theses expressions into Eqs. (1), and keeping terms of order $O\left(\sqrt{N_{E, I}}\right)$, we obtain the following dynamics for the LFPs fluctuations:

$$
\begin{aligned}
& \frac{d \tilde{V}_{E}^{1}}{d t}=A_{E E}^{1} \tilde{V}_{E}^{1}(t)+A_{E I}^{1} \tilde{V}_{I}^{1}(t)+C_{E E}^{12} \tilde{V}_{E}^{2}(t-\tau)+\sigma_{E_{1}} \xi_{E_{1}}(t) \\
& \frac{d \tilde{V}_{I}^{1}}{d t}=A_{I E}^{1} \tilde{V}_{E}^{1}(t)+A_{I I}^{1} \tilde{V}_{I}^{1}(t)+C_{I E}^{12} \tilde{V}_{E}^{2}(t-\tau)+\sigma_{I_{1}} \xi_{I_{1}}(t) \\
& \frac{d \tilde{V}_{E}^{2}}{d t}=A_{E E}^{2} \tilde{V}_{E}^{2}(t)+A_{E I}^{2} \tilde{V}_{I}^{2}(t)+C_{E E}^{21} \tilde{V}_{E}^{1}(t-\tau)+\sigma_{E_{2}} \xi_{E_{2}}(t) \\
& \frac{d \tilde{V}_{I}^{2}}{d t}=A_{I E}^{2} \tilde{V}_{E}^{2}(t)+A_{I I}^{2} \tilde{V}_{I}^{2}(t)+C_{I E}^{21} \tilde{V}_{E}^{1}(t-\tau)+\sigma_{I_{2}} \xi_{I_{2}}(t),
\end{aligned}
$$

where $\xi_{k}(t), k=E_{1}, I_{1}, E_{2}, I_{2}$ are independent gaussian white noises and the parameters of each network are given by

$$
\begin{aligned}
A_{E E}^{1} & =-\alpha_{E}-\beta_{E} f\left(s_{E_{10}}\right)+\left(1-E_{10}\right) \beta_{E} f^{\prime}\left(s_{E_{10}}\right) W_{E E}, \\
A_{E I}^{1} & =-c_{E I}\left(1-E_{10}\right) \beta_{E} f^{\prime}\left(s_{E_{10}}\right) W_{E I}, \\
A_{I E}^{1} & =c_{I E}\left(1-I_{10}\right) \beta_{I} f^{\prime}\left(s_{I_{10}}\right) W_{I E}, \\
A_{I I}^{1} & =-\alpha_{I}-\beta_{I} f\left(s_{I_{10}}\right)-\left(1-I_{10}\right) \beta_{I} f^{\prime}\left(s_{I_{10}}\right) W_{I I}, \\
A_{E E}^{2} & =-\alpha_{E}-\beta_{E} f\left(s_{E_{20}}\right)+\left(1-E_{20}\right) \beta_{E} f^{\prime}\left(s_{E_{20}}\right) W_{E E}, \\
A_{E I}^{2} & =-c_{E I}\left(1-E_{20}\right) \beta_{E} f^{\prime}\left(s_{E_{20}}\right) W_{E I}, \\
A_{I E}^{2} & =c_{I E}\left(1-I_{20}\right) \beta_{I} f^{\prime}\left(s_{I_{20}}\right) W_{I E}, \\
A_{I I}^{2} & =-\alpha_{I}-\beta_{I} f\left(s_{I_{20}}\right)-\left(1-I_{20}\right) \beta_{I} f^{\prime}\left(s_{I_{20}}\right) W_{I I},
\end{aligned}
$$

and

$$
\begin{array}{ll}
\sigma_{E_{1}}=\sqrt{2 \alpha_{E} E_{10}}, & \sigma_{I_{1}}=\sqrt{2 \alpha_{I} I_{10}}, \\
\sigma_{E_{2}}=\sqrt{2 \alpha_{E} E_{20}}, & \sigma_{I_{2}}=\sqrt{2 \alpha_{I} I_{20}}, \\
c_{E I}=\sqrt{N_{E} / N_{I}}, & c_{I E}=\sqrt{N_{I} / N_{E}} .
\end{array}
$$


The effective couplings are

$$
\begin{aligned}
& C_{E E}^{12}=\left(1-E_{10}\right) \beta_{E} f^{\prime}\left(s_{E_{10}}\right) L_{E E}^{12}, \\
& C_{I E}^{12}=c_{I E}\left(1-I_{10}\right) \beta_{I} f^{\prime}\left(s_{I_{10}}\right) L_{I E}^{12}, \\
& C_{E E}^{21}=\left(1-E_{20}\right) \beta_{E} f^{\prime}\left(s_{E_{20}}\right) L_{E E}^{21}, \\
& C_{I E}^{21}=c_{I E}\left(1-I_{20}\right) \beta_{I} f^{\prime}\left(s_{I_{20}}\right) L_{I E}^{21} .
\end{aligned}
$$

This system of four linear equations sustained by noise is a good representation of the dynamics of excitatory and inhibitory LFPs in the quasi-cycle regime. This can be seen as a system of two connected networks of excitatory and inhibitory populations with effective intrapopulation connectivity coefficients $A_{E E}^{i}, A_{E I}^{i}, A_{I E}^{i}$, and $A_{I I}^{i}, i=1,2$. The noise intensities in the $\mathrm{E}$ and I populations are, respectively, $\sigma_{E_{i}}$ and $\sigma_{I_{i}}, i=1,2$. The effective long-range excitatory connections from the first network to the $\mathrm{E}$ and I populations of the second network are $C_{E E}^{21}$ and $C_{I E}^{21}$, respectively, while the effective long-range excitatory connections from the second network to the excitatory and inhibitory populations of the first network are respectively $C_{E E}^{12}$ and $C_{I E}^{12}$. Following the expressions of the effective long-range excitatory connections $C_{E E}^{i j}$ and $C_{I E}^{i j}, i \neq$ $j=1,2$, the two networks have effective connectivities that are different from the anatomical connectivity since $C_{E E}^{i j} \neq$ $L_{E E}^{i j}$ and $C_{I E}^{i j} \neq L_{I E}^{i j}$. Moreover, $C_{E E}^{12} \neq C_{E E}^{21}$ and $C_{I E}^{12} \neq C_{E E}^{21}$ for the asymmetric and heterogeneous cases.

\section{APPENDIX B: LINEAR STABILITY}

In the quasi-cycle regime, the dynamics of the LFPs converge to zero in the absence of noise. However, we can still extract important properties from the deterministic decay dynamics. Here, we investigate the properties of the decay dynamics in the absence of noise. The dynamics of such decays are given by $\left(\sigma_{E_{1}}=\sigma_{E_{2}}=\sigma_{I_{1}}=\sigma_{I_{2}}=0\right)$

$$
\begin{aligned}
& \frac{d \tilde{V}_{E}^{1}(t)}{d t}=A_{E E}^{1} \tilde{V}_{E}^{1}(t)+A_{E I}^{1} \tilde{V}_{I}^{1}(t)+C_{E E}^{12} \tilde{V}_{E}^{2}(t-\tau), \\
& \frac{d \tilde{V}_{I}^{1}(t)}{d t}=A_{I E}^{1} \tilde{V}_{E}^{1}(t)+A_{I I}^{1} \tilde{V}_{I}^{1}(t)+C_{I E}^{12} \tilde{V}_{E}^{2}(t-\tau), \\
& \frac{d \tilde{V}_{E}^{2}(t)}{d t}=A_{E E}^{2} \tilde{V}_{E}^{2}(t)+A_{E I}^{2} \tilde{V}_{I}^{2}(t)+C_{E E}^{21} \tilde{V}_{E}^{1}(t-\tau), \\
& \frac{d \tilde{V}_{I}^{2}(t)}{d t}=A_{I E}^{2} \tilde{V}_{E}^{2}(t)+A_{I I}^{2} \tilde{V}_{I}^{2}(t)+C_{I E}^{21} \tilde{V}_{E}^{1}(t-\tau) .
\end{aligned}
$$

We look for solutions of these equations in the form of exponential decays:

$$
\begin{array}{ll}
\tilde{V}_{E}^{1}(t)=\tilde{A}_{E}^{1} \exp (\lambda t), & \tilde{V}_{I}^{1}(t)=\tilde{A}_{I}^{1} \exp (\lambda t), \\
\tilde{V}_{E}^{2}(t)=\tilde{A}_{E}^{2} \exp (\lambda t), & \tilde{V}_{I}^{2}(t)=\tilde{A}_{I}^{2} \exp (\lambda t) .
\end{array}
$$

Replacing these expressions into the noise-free dynamics [Eqs. (B1)] above yields the following relationships:

$$
\begin{aligned}
& \tilde{\alpha}_{I E}^{1}=\frac{\tilde{A}_{I}^{1}}{\tilde{A}_{E}^{1}}=\frac{A_{I E}^{1} C_{E E}^{12}-C_{I E}^{12}\left(A_{E E}^{1}-\lambda\right)}{A_{E I}^{1} C_{I E}^{12}-C_{E E}^{12}\left(A_{I I}^{1}-\lambda\right)}, \\
& \tilde{\alpha}_{E}^{21}=\frac{\tilde{A}_{E}^{2}}{\tilde{A}_{E}^{1}}=\frac{\left(A_{E E}^{1}-\lambda\right)\left(A_{I I}^{1}-\lambda\right)-A_{I E}^{1} A_{E I}^{1}}{A_{E I}^{1} C_{I E}^{12}-C_{E E}^{12}\left(A_{I I}^{1}-\lambda\right)} \exp (\lambda \tau),
\end{aligned}
$$

$$
\begin{aligned}
& \tilde{\alpha}_{I E}^{2}=\frac{\tilde{A}_{I}^{2}}{\tilde{A}_{E}^{2}}=\frac{A_{I E}^{2} C_{E E}^{21}-C_{I E}^{21}\left(A_{E E}^{2}-\lambda\right)}{A_{E I}^{2} C_{I E}^{21}-C_{E E}^{21}\left(A_{I I}^{2}-\lambda\right)}, \\
& \tilde{\alpha}_{E}^{12}=\frac{\tilde{A}_{E}^{1}}{\tilde{A}_{E}^{2}}=\frac{\left(A_{E E}^{2}-\lambda\right)\left(A_{I I}^{2}-\lambda\right)-A_{I E}^{2} A_{E I}^{2}}{A_{E I}^{2} C_{I E}^{21}-C_{E E}^{21}\left(A_{I I}^{2}-\lambda\right)} \exp (\lambda \tau) .
\end{aligned}
$$

Using the relation $\tilde{\alpha}_{E}^{12}=\left(\tilde{\alpha}_{E}^{21}\right)^{-1}$ we obtain the following characteristic equation:

$$
\begin{aligned}
{\left[\left(A_{I I}^{2}-\lambda\right)\left(A_{E E}^{2}-\lambda\right)-A_{E I}^{2} A_{I E}^{2}\right] } \\
\quad \times\left[\left(A_{I I}^{1}-\lambda\right)\left(A_{E E}^{1}-\lambda\right)-A_{E I}^{1} A_{I E}^{1}\right] \\
=e^{-2 \lambda \tau}\left[A_{E I}^{1} C_{I E}^{12}-C_{E E}^{12}\left(A_{I I}^{1}-\lambda\right)\right] \\
\quad \times\left[A_{E I}^{2} C_{I E}^{21}-C_{E E}^{21}\left(A_{I I}^{2}-\lambda\right)\right] .
\end{aligned}
$$

The value of the amplitude ratio can be obtained by first solving the characteristic equation. The solution of interest is the eigenvalue with the largest real part (note that this real part should be negative since we are in the quasi-cycle regime). Replacing this particular eigenvalue in the expression of the amplitude ratio above allows us to obtain the right expression for these ratios. Note that the ratios are complex quantities and can therefore be put in exponential form, with the argument of the exponential being the phase difference and its modulus the real amplitude ratio. We then obtain the amplitude ratio and phase difference between the inhibitory and excitatory LFPs in each network as

$$
\begin{array}{cc}
\alpha_{1}=\operatorname{abs}\left(\tilde{\alpha}_{I E}^{1}\right), & \alpha_{2}=\operatorname{abs}\left(\tilde{\alpha}_{I E}^{2}\right), \\
\delta_{1}=\arg \left(\tilde{\alpha}_{I E}^{1}\right), & \delta_{2}=\arg \left(\tilde{\alpha}_{I E}^{2}\right),
\end{array}
$$

and the phase difference between excitatory populations of different networks ( 1 and 2 , respectively) as

$$
\Delta \phi=\arg \left(\tilde{\alpha}_{E}^{12}\right) .
$$

Similar analysis was done for the case of purely inhibitory populations (not shown here) to compute the deterministic phase difference in solid black dots [Fig. 7(a)].

\section{APPENDIX C: STOCHASTIC AVERAGING METHOD (SAM) AND ENVELOPE-PHASE DECOMPOSITION}

We are interested in the fluctuations from the baseline activities (LNA): $\tilde{V}_{E}^{i}(t)=c_{E}\left(E_{i}(t)-E_{i 0}\right) ; \quad \tilde{V}_{I}^{i}(t)=c_{I}\left(I_{i}(t)-\right.$ $I_{i 0}$ ), where $E_{i 0}$ and $I_{i 0}$ are the deterministic fixed point activities. We are further interested in the slow envelope and phase dynamics of the fluctuations. For that, we seek solutions in the form of Eqs. (4) and plug these expressions in the equations for the LFPs, Eqs. (A2). This yields a system of differential equations in terms of $Z_{1}, Z_{2}, \phi_{1}$, and $\phi_{2}$ as follows:

$$
\begin{aligned}
& \dot{Z}_{1}(t)=F_{1}^{1}\left(Z_{1,2}, \phi_{1,2}\right)+G_{1}^{1}\left(Z_{1,2}, \phi_{1,2}, \xi_{E_{1,2}}, \xi_{I_{1,2}}\right), \\
& \dot{\phi}_{1}(t)=F_{2}^{1}\left(Z_{1,2}, \phi_{1,2}\right)+G_{2}^{1}\left(Z_{1,2}, \phi_{1,2}, \xi_{E_{1,2}}, \xi_{I_{1,2}}\right), \\
& \dot{Z}_{2}(t)=F_{1}^{2}\left(Z_{1,2}, \phi_{1,2}\right)+G_{1}^{2}\left(Z_{1,2}, \phi_{1,2}, \xi_{E_{1,2}}, \xi_{I_{1,2}}\right), \\
& \dot{\phi}_{2}(t)=F_{2}^{2}\left(Z_{1,2}, \phi_{1,2}\right)+G_{2}^{2}\left(Z_{1,2}, \phi_{1,2}, \xi_{E_{1,2}}, \xi_{I_{1,2}}\right),
\end{aligned}
$$


where

$$
\begin{aligned}
& F_{1}^{1}\left(Z_{1,2}, \phi_{1,2}\right)=\frac{1}{\alpha_{1} \sin \left(\delta_{1}\right)}\left[\alpha_{1} f_{1}^{1}\left(Z_{1,2}, \phi_{1,2}\right) \sin \left(\omega_{0} t+\phi_{1}(t)+\delta_{1}\right)-f_{2}^{1}\left(Z_{1,2}, \phi_{1,2}\right) \sin \left(\omega_{0} t+\phi_{1}(t)\right)\right] \\
& F_{2}^{1}\left(Z_{1,2}, \phi_{1,2}\right)=\frac{1}{\alpha_{1} Z_{1} \sin \left(\delta_{1}\right)}\left[\alpha_{1} f_{1}^{1}\left(Z_{1,2}, \phi_{1,2}\right) \cos \left(\omega_{0} t+\phi_{1}(t)+\delta_{1}\right)-f_{2}^{1}\left(Z_{1,2}, \phi_{1,2}\right) \cos \left(\omega_{0} t+\phi_{1}(t)\right)\right] \text {, } \\
& F_{1}^{2}\left(Z_{1,2}, \phi_{1,2}\right)=\frac{1}{\alpha_{2} \sin \left(\delta_{2}\right)}\left[\alpha_{2} f_{1}^{2}\left(Z_{1,2}, \phi_{1,2}\right) \sin \left(\omega_{0} t+\phi_{2}(t)+\delta_{2}\right)-f_{2}^{2}\left(Z_{1,2}, \phi_{1,2}\right) \sin \left(\omega_{0} t+\phi_{2}(t)\right)\right], \\
& F_{2}^{2}\left(Z_{1,2}, \phi_{1,2}\right)=\frac{1}{\alpha_{2} Z_{2} \sin \left(\delta_{2}\right)}\left[\alpha_{2} f_{1}^{2}\left(Z_{1,2}, \phi_{1,2}\right) \cos \left(\omega_{0} t+\phi_{2}(t)+\delta_{2}\right)-f_{2}^{2}\left(Z_{1,2}, \phi_{1,2}\right) \cos \left(\omega_{0} t+\phi_{2}(t)\right)\right], \\
& G_{1}^{1}\left(Z_{1,2}, \phi_{1,2}, \xi_{E_{1,2}}, \xi_{I_{1,2}}\right)=\frac{1}{\alpha_{1} \sin \left(\delta_{1}\right)}\left[\alpha_{1} g_{1}^{1}\left(Z_{1,2}, \phi_{1,2}, \xi_{E_{1,2}}, \xi_{I_{1,2}}\right) \sin \left(\omega_{0} t+\phi_{1}(t)+\delta_{1}\right)\right. \\
& \left.-g_{2}^{1}\left(Z_{1,2}, \phi_{1,2}, \xi_{E_{1,2}}, \xi_{I_{1,2}}\right) \sin \left(\omega_{0} t+\phi_{1}(t)\right)\right] \\
& G_{2}^{1}\left(Z_{1,2}, \phi_{1,2}, \xi_{E_{1,2}}, \xi_{I_{1,2}}\right)=\frac{1}{\alpha_{1} Z_{1} \sin \left(\delta_{1}\right)}\left[\alpha_{1} g_{1}^{1}\left(Z_{1,2}, \phi_{1,2}, \xi_{E_{1,2}}, \xi_{I_{1,2}}\right) \cos \left(\omega_{0} t+\phi_{1}(t)+\delta_{1}\right)\right. \\
& \left.-g_{2}^{1}\left(Z_{1,2}, \phi_{1,2}, \xi_{E_{1,2}}, \xi_{I_{1,2}}\right) \cos \left(\omega_{0} t+\phi_{1}(t)\right)\right] \\
& G_{1}^{2}\left(Z_{1,2}, \phi_{1,2}, \xi_{E_{1,2}}, \xi_{I_{1,2}}\right)=\frac{1}{\alpha_{2} \sin \left(\delta_{2}\right)}\left[\alpha_{2} g_{1}^{2}\left(Z_{1,2}, \phi_{1,2}, \xi_{E_{1,2}}, \xi_{I_{1,2}}\right) \sin \left(\omega_{0} t+\phi_{2}(t)+\delta_{2}\right)\right. \\
& \left.-g_{2}^{2}\left(Z_{1,2}, \phi_{1,2}, \xi_{E_{1,2}}, \xi_{I_{1,2}}\right) \sin \left(\omega_{0} t+\phi_{2}(t)\right)\right] \\
& G_{2}^{2}\left(Z_{1,2}, \phi_{1,2}, \xi_{E_{1,2}}, \xi_{I_{1,2}}\right)=\frac{1}{\alpha_{2} Z_{2} \sin \left(\delta_{2}\right)}\left[\alpha_{2} g_{1}^{2}\left(Z_{1,2}, \phi_{1,2}, \xi_{E_{1,2}}, \xi_{I_{1,2}}\right) \cos \left(\omega_{0} t+\phi_{2}(t)+\delta_{2}\right)\right. \\
& \left.-g_{2}^{2}\left(Z_{1,2}, \phi_{1,2}, \xi_{E_{1,2}}, \xi_{I_{1,2}}\right) \cos \left(\omega_{0} t+\phi_{2}(t)\right)\right] \text {, } \\
& f_{1}^{1}\left(Z_{1,2}, \phi_{1,2}\right)=\omega_{0} Z_{1}(t) \sin \left(\omega_{0} t+\phi_{1}(t)\right)+A_{E E}^{1} Z_{1} \cos \left(\omega_{0} t+\phi_{1}(t)\right)+A_{E I}^{1} \alpha_{1} Z_{1} \cos \left(\omega_{0} t+\phi_{1}(t)+\delta_{1}\right) \\
& +C_{E E}^{12} Z_{2}(t-\tau) \cos \left(\omega_{0} t-\omega_{0} \tau+\phi_{2}(t-\tau)\right), \\
& f_{2}^{1}\left(Z_{1,2}, \phi_{1,2}\right)=\alpha_{1} \omega_{0} Z_{1}(t) \sin \left(\omega_{0} t+\phi_{1}(t)+\delta_{1}\right)+A_{I E}^{1} Z_{1} \cos \left(\omega_{0} t+\phi_{1}(t)\right)+A_{I I}^{1} \alpha_{1} Z_{1} \cos \left(\omega_{0} t+\phi_{1}(t)+\delta_{1}\right) \\
& +C_{I E}^{12} Z_{2}(t-\tau) \cos \left(\omega_{0} t-\omega_{0} \tau+\phi_{2}(t-\tau)\right), \\
& f_{1}^{2}\left(Z_{1,2}, \phi_{1,2}\right)=\omega_{0} Z_{2}(t) \sin \left(\omega_{0} t+\phi_{2}(t)\right)+A_{E E}^{2} Z_{2} \cos \left(\omega_{0} t+\phi_{2}(t)\right)+A_{E I}^{2} \alpha_{2} Z_{2} \cos \left(\omega_{0} t+\phi_{2}(t)+\delta_{2}\right) \\
& +C_{E E}^{21} Z_{1}(t-\tau) \cos \left(\omega_{0} t-\omega_{0} \tau+\phi_{1}(t-\tau)\right), \\
& f_{2}^{2}\left(Z_{1,2}, \phi_{1,2}\right)=\alpha_{2} \omega_{0} Z_{2}(t) \sin \left(\omega_{0} t+\phi_{2}(t)+\delta_{2}\right)+A_{I E}^{2} Z_{2} \cos \left(\omega_{0} t+\phi_{2}(t)\right)+A_{I I}^{2} \alpha_{2} Z_{2} \cos \left(\omega_{0} t+\phi_{2}(t)+\delta_{2}\right) \\
& +C_{I E}^{21} Z_{1}(t-\tau) \cos \left(\omega_{0} t-\omega_{0} \tau+\phi_{1}(t-\tau)\right), \\
& g_{1}^{1}\left(Z_{1,2}, \phi_{1,2}, \xi_{E_{1,2}}, \xi_{I_{1,2}}\right)=\sigma_{E_{1}} \xi_{E_{1}}(t), \quad g_{2}^{1}\left(Z_{1,2}, \phi_{1,2}, \xi_{E_{1,2}}, \xi_{I_{1,2}}\right)=\sigma_{I_{1}} \xi_{I_{1}}(t), \\
& g_{1}^{2}\left(Z_{1,2}, \phi_{1,2}, \xi_{E_{1,2}}, \xi_{I_{1,2}}\right)=\sigma_{E_{2}} \xi_{E_{2}}(t), \quad g_{2}^{2}\left(Z_{1,2}, \phi_{1,2}, \xi_{E_{1,2}}, \xi_{I_{1,2}}\right)=\sigma_{I_{2}} \xi_{I_{2}}(t) .
\end{aligned}
$$

The envelope and phase dynamics described by Eqs. (C1) are hard to visualize and analyze. To simplify their dynamics, we apply the stochastic averaging method (SAM). The SAM states that, under certain conditions (usually met for regular functions like $F_{1}^{i}, F_{2}^{i}$ and $G_{1}^{i}, G_{2}^{i}, i=1,2$ ), the above system of four stochastic differential equations can be approximated by the following four-dimensional Markov process [27]:

$$
d X(t)=m(X) d t+h(X) d W(t)
$$

where $X(t)=\left[Z_{1}(t) ; \phi_{1}(t) ; Z_{2}(t) ; \phi_{2}(t)\right]^{\prime}, m$ is a $4 \times 1$ matrix, $h$ is a $4 \times 4$ matrix, and $W(t)$ denotes a four-dimensional vector of independent Wiener processes with unit variance. Also, $m$ and $h$ are respectively $O\left(\epsilon^{2}\right)$ and $O(\epsilon)$ functions defined as

$$
\begin{aligned}
m & =T^{a v}\left(E\{F\}+\int_{-\infty}^{0} E\left\{\left(\frac{\partial G}{\partial X}\right)_{t}(G)_{t+z}\right\} d z\right), \\
h h^{\prime} & =T^{a v}\left(\int_{-\infty}^{\infty} E\left\{(G)_{t}\left(G^{\prime}\right)_{t+z}\right\} d z\right),
\end{aligned}
$$


where $\epsilon$ is a small parameter which can be assumed proportional to the real part of the most unstable eigenvalue. Here $\left(^{\prime}\right)$ denotes transposition, and $\left(\frac{\partial G}{\partial X}\right)_{t}$ is a $4 \times 4$ Jacobian matrix. Moreover, $E$ denotes the expectation operator and $T^{a v}$ is the time-averaging operator defined by

$$
T^{a v}()=\frac{1}{T_{0}} \int_{t 0}^{t 0+T 0}() d t,
$$

where $T_{0}=\frac{2 \pi}{\omega_{0}}$ is the period of an oscillation cycle. When evaluating the expectations in the stochastic averages formula, the elements of $X$ are treated as constants. The result of these calculations leads to the coupled envelope-phase dynamics in Eqs. (5).

\section{APPENDIX D: DIP STATISTIC AND PHASE-LOCKING VALUE}

The dip value is a measure of multimodality of a given time series. It computes the maximum difference, over all points of the time series, between the empirical distribution and the unimodal distribution that minimizes that maximum difference. The uniform distribution is chosen as the unimodal distribution when performing the dip test for multimodality [37]. The dip value approaches asymptotically zero for a unimodal distribution and a positive constant for a multimodal distribution. We used a MATLAB version of the program already written by the authors [38] to produce Fig. 5(a). The increase of the dip value as the delay increases is a signature of the multimodality of the phase difference time series. In our case, this multimodality is a bimodality corresponding to the two symmetric out-of-phase states present. The dip test therefore confirms the presence of OPL.

To measure the strength of phase synchronization between the two networks, we also computed the phase-locking value (PLV) [11,12] as the delay varies. The PLV is a quantity to measure the strength of the phase synchronization and is defined as

$$
\mathrm{PLV}=\frac{1}{n}\left|\sum_{k=1}^{n} e^{-j \Delta \phi_{k}}\right|,
$$

where $j=\sqrt{-1}, n$ is the total number of points, and $\Delta \phi_{k}=$ $\Delta \phi\left(t_{k}\right)=\phi_{1}\left(t_{k}\right)-\phi_{2}\left(t_{k}\right)$. The PLV is computed in Fig. 5(b) and captures well the transition between unimodal and bimodal behavior in the phase difference.
[1] A. Pikovsky, M. Rosenblum, and J. Kurths, Synchronization, 1st ed. (Cambridge University Press, Cambridge, U.K., 2001).

[2] J. A. Acebron, L. Bonilla, C. J. Perez Vicente, F. Ritort, and R. Spigler, Rev. Mod. Phys. 77, 137 (2005).

[3] U. Thounaojam, J. Cui, S. Norman, R. Butera, and C. Canavier, PLoS Comput. Biol. 10, e1003622 (2014).

[4] J. Hizanidis, A. Balanov, A. Amann, and E. Schöll, Int. J. Bifurcation Chaos 16, 1701 (2006).

[5] P. C. Bressloff, Phys. Rev. E 82, 051903 (2010).

[6] A. J. McKane and T. J. Newman, Phys. Rev. Lett. 94, 218102 (2005).

[7] B. Sancristobal, B. Rebollo, P. Boada, M. V. Sanchez-Vives, and J. Garcia-Ojalvo, Nat. Phys. 12, 881 (2016).

[8] A. Palmigiano, T. Geisel, F. Wolf, and D. Battaglia, Nat. Neurosci. 20, 1014 (2017).

[9] B. Doiron, B. Lindner, A. Longtin, L. Maler, and J. Bastian, Phys. Rev. Lett. 93, 048101 (2004).

[10] E. Ledoux and N. Brunel, Front. Comput. Neurosci. 5, 25 (2011).

[11] F. Varela, J.-P. Lachaux, E. Rodriguez, and J. Martinerie, Nat. Rev. Neurosci. 2, 229 (2001).

[12] E. Lowet, M. J. Roberts, A. Peter, B. Gips, and P. De Weerd, eLife 6, e26642 (2017).

[13] D. Battaglia, N. Brunel, and D. Hansel, Phys. Rev. Lett. 99, 238106 (2007).

[14] G. Dumont and B. Gutkin, PLoS Comput. Biol. 15, e1007019 (2019).

[15] D. Battaglia, A. Witt, F. Wolf, and T. Geisel, PLoS Comput. Biol. 8, e1002438 (2012).

[16] C. Kirst, M. Timme, and D. Battaglia, Nat. Commun. 7, 11061 (2016).

[17] A. Witt, A. Palmigiano, A. Neef, A. El Hady, F. Wolf, and D. Battaglia, Front. Neural Circ. 7, 49 (2013).

[18] D. Xing, Y. Shen, S. Burns, C.-I. Yeh, R. Shapley, and W. Li, J. Neurosci. 32, 13873 (2012).
[19] A. S. Powanwe and A. Longtin, Sci. Rep. 9, 1 (2019).

[20] R. M. Hutchison, T. Womelsdorf, E. A. Allen, P. A. Bandettini, V. D. Calhoun, M. Corbetta, S. Della Penna, J. H. Duyn, G. H. Glover, J. Gonzalez-Castillo et al., Neuroimage 80, 360 (2013).

[21] R. M. Hutchison, T. Womelsdorf, J. S. Gati, S. Everling, and R. S. Menon, Hum. Brain Mapp. 34, 2154 (2013).

[22] J. Gonzalez-Castillo and P. A. Bandettini, Neuroimage 180, 526 (2018).

[23] P. Fries, Trends Cognit. Sci. 9, 474 (2005).

[24] P. Fries, Neuron 88, 220 (2015).

[25] A. M. Bastos, J. Vezoli, and P. Fries, Curr. Opin. Neurobiol. 31, 173 (2015).

[26] E. Wallace, M. Benayoun, W. Van Drongelen, and J. D. Cowan, PLoS One 6, e14804 (2011).

[27] J. Roberts and P. Spanos, Int. J. Non-Linear Mech. 21, 111 (1986).

[28] P. E. Greenwood, M. D. McDonnell, and L. M. Ward, J. Coupled Syst. Multiscale Dyn. 4, 1 (2016).

[29] Y. Kyrychko, K. Blyuss, and E. Schöll, Philos. Trans. R. Soc. A 371, 20120466 (2013).

[30] A. Longtin, Phys. Rev. A 44, 4801 (1991).

[31] G. Dumont, G. Northoff, and A. Longtin, Phys. Rev. E 90 012702 (2014).

[32] S. Keeley, A. A. Fenton, and J. Rinzel, J. Neurophysiol. 117, 950 (2017).

[33] N. G. Van Kampen, Stochastic Processes in Physics and Chemistry (Elsevier, Amsterdam, 1992), Vol. 1.

[34] T. Ohira and J. D. Cowan, in Mathematics of Neural Networks (Springer, Berlin, 1997), pp. 290-294.

[35] P. C. Bressloff, SIAM J. Appl. Math. 70, 1488 (2010).

[36] M. Benayoun, J. D. Cowan, W. van Drongelen, and E. Wallace, PLoS Comput. Biol. 6, e1000846 (2010).

[37] J. A. Hartigan and P. M. Hartigan, Ann. Stat. 13, 70 (1985).

[38] P. M. Hartigan, J. R. Stat. Soc. Ser. C (Appl. Stat.) 34, 320 (1985). 